\title{
Other-Cell Interference Aware Precoding for the Downlink of Multi-User MIMO AF Communication
}

\author{
Fabien Héliot, Usama Asif, Reza Hoshyar, and Rahim Tafazolli \\ Centre for Communication Systems Research, University of Surrey, \\ Guildford GU2 7HX, UK, Email: F.Heliot@Surrey.ac.uk
}

\begin{abstract}
Amplify-and-forward (AF) is one of the most popular and simple approaches for transmitting information over a cooperative multi-input multi-output (MIMO) relay channel. In cooperative communication, relays are employed for improving the coverage or enhancing the spectral efficiency, especially of cell-edge users. However, in a multi-cell context, the use of relays will also lead to an increase in interferences that are experienced by cell-edge users of neighboring cells. In this paper, two novel precoding schemes for mitigating this adverse effect of cooperative communication are proposed. They are designed by taking into account the effect of interference coming from neighboring cells, i.e. other cell-interference (OCI), for maximizing the sumrate of cell-edge users. Our novel OCI-aware precoding schemes are compared against non OCI-aware precoding techniques and results show the large performance gain in terms of sum-rate that our schemes can achieved especially for large numbers of users and/or antennas in the multi-cell system.
\end{abstract}

\section{INTRODUCTION}

Cooperative communication has recently attracted considerable research interests [1]-[3]. Amplify-and-forward (AF) is a simple and practical approach for implementing cooperative multi-input multi-output (MIMO) communication. In AF, the relay node $(\mathrm{RN})$ simply amplifies the received signal from the source node $(\mathrm{SN})$ and forwards it to the destination node (DN). In the original AF scheme [3], the RN was first used as a simple equal gain amplifier. Since then, it has been shown in [4] and other many works that the RN can also be utilized as a smart precoder for improving the spectral efficiency of single and multi-user (MU) MIMO AF communication.

As far as the downlink (DL) of MU MIMO AF communication is concerned, some methods have first been proposed in [5] and [6] to efficiently perform the precoding at the $\mathrm{RN}$ but only for the single antenna per user case. Recently in [7], a method for the MIMO case has been designed by assuming that the full channel state information (CSI) of the relay channel is available at the $\mathrm{SN}$ and that dirty paper coding is employed. Then in [8], we have developed three precoding methods for the DL of MU MIMO AF system, namely the AF-statistical knowledge of the relay-destination links, AF-channel block diagonalization (CBD) and AF-constrained gradient search for DL (CGSDL) methods, by considering two more realistic CSI assumptions than in [7], i.e. only the receive CSI or both receive and transmit CSI is available at the RN and, hence, without relying on DPC at the SN. All the previously cited precoding techniques only considered MU interference from different users within the same cell and, thus, did not take into account OCI. However, it has been shown in [9] and [10] that interference coming from neighboring cells significantly degrade the cell-edge user and overall sumrate performances in cellular networks. Consequently, an OCIaware precoding technique has been proposed in [11] for mitigating the effect of OCI in point-to-point (P2P) multi-cell communication. In cooperative multi-cell communication, the OCI problem will be exacerbated since one induced effect of relaying is the increase of OCI for neighboring cell-edge users, which justify the need for proper OCI mitigation.

In this paper, we extend our work in [8], which has been undertaken for the single cell scenario, to the cooperative MU multi-cell scenario by incorporating the effect of OCI in our precoding structure at the $\mathrm{RN}$. We model the DL of the MU MIMO AF system in presence of OCI in Section II and derive its sum-rate expression, which is used as a design criterion for our novel precoding schemes that are presented in Section III. Our AF-enhanced CBD (ECBD) and AF-enhanced CGSDL (ECGSDL) schemes are designed for maximizing this criterion by considering that the SN-RN and RN-DN link CSI and the interference plus noise covariance matrix of each cell-edge user are available at the RN. The sum-rate performances of our OCI-aware schemes are presented in Section IV and compared against those of the schemes of [8] in presence of OCI. Results indicate that a large sum-rate gain can be obtained by using our OCI-aware techniques, especially for large numbers of users and/or antennas. Finally, conclusions are drawn in Section V.

\section{MU MiMO AF System Model in Presence of OCI}

We consider a MU MIMO AF system that is composed of $K+2$ nodes, i.e. a $\mathrm{SN}$ with $n$ antennas, a nonregenerative $\mathrm{RN}$ with $q$ antennas and $K$ DNs with $r_{k}$ antennas, as it is depicted in Fig. 1. In addition, we assume that the direct link is weak in comparison with the relay link and, thus, it is omitted.

For the simplicity of the introduction, we assume a half duplex relaying scenario with two equal duration phases as in [4], where in the first phase the $\mathrm{SN}$ broadcasts the signal $\mathbf{x}=\sum_{k=1}^{K} \mathbf{R}_{k} \mathbf{s}_{k}$ to the $\mathrm{RN}$, and in the second phase the $\mathrm{RN}$ transmits to the DN. Note that $\mathbf{R}_{k} \in \mathbb{C}^{n \times n}$ is the $k$-th user precoding matrix at the $\mathrm{SN}$ and $\mathbf{s}_{k} \in \mathbb{C}^{n \times 1}$, which we

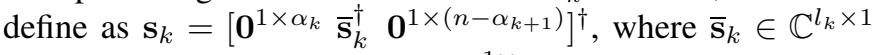
is the $k$-th message of length $l_{k}, \mathbf{0}^{1 \times \alpha_{k}}$ is an all zero vector of length $\alpha_{k}$ and $\alpha_{k}=\sum_{j=1}^{k-1} l_{j}$. Consequently, $\mathrm{E}\left\{\mathbf{s}_{k} \mathbf{s}_{k}^{\dagger}\right\}=\mathbf{I}_{l_{k}}$, where $\mathbf{I}_{l_{k}}$ is a $l_{k} \times l_{k}$ identity matrix and $\mathrm{E}\{$.$\} stands for$ the expectation. In addition, we define $l$ as $l=\sum_{k=1}^{K} l_{k}$ and assume that $l_{k} \leq r_{k}$ and $l \leq n$. 




Fig. 1. DL of MU MIMO AF system in presence of OCI.

The signal $\mathbf{x}$ is received by the $\mathrm{RN}$ as $\mathbf{y}_{1}=\mathbf{H}_{1} \mathbf{x}+\mathbf{n}_{1}$ at the end of the first phase, where $\mathbf{H}_{1} \in \mathbb{C}^{q \times n}$ characterize the MIMO channel of the SN-RN link. During the second phase, the signal $\mathbf{y}_{1}$ is amplified by using the precoding matrix $\mathbf{G} \in \mathbb{C}^{q \times q}$, is then transmitted towards the DNs and is received as $\mathbf{y}_{2, k}=\mathbf{H}_{2, k} \mathbf{G y}_{1}+\mathbf{n}_{2, k}$ by the $k$-th $\mathrm{DN}$, where $\mathbf{H}_{2, k} \in \mathbb{C}^{r_{k} \times q}$ characterizes the MIMO channel of the $k$-th RN-DN link. Moreover, each of the channel matrices $\mathbf{H}_{1}$ and $\mathbf{H}_{2, k}$ is a random matrix having independent and identically distributed (i.i.d.) complex circular Gaussian entries with zero-mean and unit variance. Furthermore, $\mathbf{n}_{1} \in \mathbb{C}^{q \times 1}$ and $\mathbf{n}_{2, k} \in \mathbb{C}^{r_{k} \times 1}$ are vectors of independent zero-mean complex Gaussian noise entries with a variance of $\sigma_{1}^{2}$ and $\sigma_{2, k}^{2}$, respectively. We consider the same multi-cell interference or OCI model as in [11], where each DN is affected by an OCI signal $\mathbf{n}_{\mathrm{I}, k}=\mathbf{H}_{\mathrm{I}, k} \mathbf{x}_{\mathrm{I}, k}$ with $\mathbf{H}_{\mathrm{I}, k} \in \mathbb{C}^{r_{k} \times n_{\mathrm{I}, k}}$ and $\mathbf{x}_{\mathrm{I}, k} \in \mathbb{C}^{n_{\mathrm{I}, k} \times 1}$ being the MIMO OCI channel and OCI signal with $n_{\mathrm{I}, k}$ co-channel interferers from the neighboring cell, respectively. At the receiver, the $k$-th estimated transmit message $\widehat{\mathbf{s}}_{k}$ is obtained such that $\widehat{\mathbf{s}}_{k}=\mathbf{S}_{k} \mathbf{y}_{2, k}$, where $\mathbf{S}_{k} \in \mathbb{C}^{n \times r_{k}}$ is the $k$-th DN postcoding matrix. Each postcoding matrix can be decomposed as $\mathbf{S}_{k}=\mathbf{T}_{k} \mathbf{U}_{k}$, where $\mathbf{T}_{k} \in \mathbb{C}^{r_{k} \times r_{k}}$ is the $k$ th user OCI suppression filter and $\mathbf{U}_{k} \in \mathbb{C}^{n \times r_{k}}$. Accordingly, the $k$-th DN received signal before and after OCI suppression can be expressed as

$$
\begin{aligned}
& \mathbf{y}_{2, k}=\mathbf{H}_{2, k} \mathbf{G H}_{1} \mathbf{x}+\mathbf{H}_{2, k} \mathbf{G n}_{1}+\mathbf{n}_{2, k}+\mathbf{n}_{\mathrm{I}, k} \\
& \mathbf{r}_{k}=\mathbf{H}_{k} \mathbf{G H}_{1} \mathbf{x}+\mathbf{H}_{k} \mathbf{G n}_{1}+\mathbf{T}_{k}\left(\mathbf{n}_{2, k}+\mathbf{n}_{\mathrm{I}, k}\right)
\end{aligned},
$$

respectively, where $\mathbf{H}_{k}=\mathbf{T}_{k} \mathbf{H}_{2, k}$. Consequently, the mutual information (MI) of each user can be expressed as [12]

$$
\begin{aligned}
I\left(\mathbf{r}_{k} ; \mathbf{s}_{k}\right)= & \frac{1}{2} \log _{2} \mid \mathbf{I}_{r_{k}}+\mathbf{H}_{k} \mathbf{G} \mathbf{H}_{1}\left(\mathbf{R}_{\mathbf{y}_{1}}-\mathbf{R}_{\mathbf{n}_{1}, k}\right) \mathbf{H}_{1}^{\dagger} \mathbf{G}^{\dagger} \mathbf{H}_{k}^{\dagger} \\
& \times\left(\mathbf{H}_{k} \mathbf{G R}_{\mathbf{n}_{1}, k} \mathbf{G}^{\dagger} \mathbf{H}_{k}^{\dagger}+\mathbf{T}_{k} \mathbf{R}_{\mathbf{n}_{\mathrm{I}}, k} \mathbf{T}_{k}^{\dagger}\right)^{-1} \mid
\end{aligned}
$$

where the factor $1 / 2$ accounts for the two-phase transmission, $\mathbf{R}_{\mathbf{y}_{1}}=\mathrm{E}\left\{\mathbf{y}_{1} \mathbf{y}_{1}^{\dagger}\right\}=\sigma_{1}^{2} \mathbf{I}_{q}+\mathbf{H}_{1} \mathbf{R}_{\mathbf{x}} \mathbf{H}_{1}^{\dagger}$ is the transmit covariance matrix, $\mathbf{R}_{\mathbf{n}_{1}, k}=\sigma_{1}^{2} \mathbf{I}_{q}+\mathbf{H}_{1}\left(\mathbf{R}_{\mathbf{x}}-\mathbf{R}_{k} \mathbf{R}_{k}^{\dagger}\right) \mathbf{H}_{1}^{\dagger}$ is the $k$-th noise plus residual intra-cell interference covariance matrix and $\mathbf{R}_{\mathbf{x}}=\sum_{j=1}^{K} \mathbf{R}_{j} \mathbf{R}_{j}^{\dagger}$. In addition, $\mathbf{R}_{\mathbf{n}_{\mathrm{I}}, k}=\sigma_{2, k}^{2} \mathbf{I}_{r_{k}}+$ $\mathbf{H}_{\mathrm{I}, k} \mathbf{R}_{\mathrm{I}, k} \mathbf{H}_{\mathrm{I}, k}^{\dagger}$ is the $k$-th noise plus OCI covariance matrix and
$\mathbf{R}_{\mathrm{I}, k}=\mathrm{E}\left\{\mathbf{x}_{\mathrm{I}, k} \mathbf{x}_{\mathrm{I}, k}^{\dagger}\right\}$. The relay link MI that is achieved by adding each user MI, i.e. sum-rate, is then simply given by

$$
\Sigma_{\mathbf{r}}=\frac{1}{2} \sum_{k=1}^{K} \log _{2}\left|\frac{\mathbf{T}_{k} \mathbf{R}_{\mathbf{n}_{\mathrm{I}}, k} \mathbf{T}_{k}^{\dagger}+\mathbf{H}_{k} \mathbf{G R}_{\mathbf{y}_{1}} \mathbf{G}^{\dagger} \mathbf{H}_{k}^{\dagger}}{\mathbf{T}_{k} \mathbf{R}_{\mathbf{n}_{\mathrm{I}}, k} \mathbf{T}_{k}^{\dagger}+\mathbf{H}_{k} \mathbf{G R}_{\mathbf{n}_{1}, k} \mathbf{G}^{\dagger} \mathbf{H}_{k}^{\dagger}}\right|,
$$

and the problem of maximizing the sum-rate under the constraint that the transmit power at the RN should not exceed $P_{2}$ can be formulated as follows

$$
\max _{\mathbf{G}} \Sigma_{\mathbf{r}} \text { s.t. } \mathbf{G} \succeq 0 ; \operatorname{tr}\left(\mathbf{G R}_{\mathbf{y}_{1}} \mathbf{G}^{\dagger}\right) \leq P_{2},
$$

where $\operatorname{tr}($.$) denotes the trace of a matrix and P_{2}$ is the total transmit power of the RN. Furthermore, notice that the optimum $\mathbf{U}_{k}$ for each user $k$ can simply be obtained as $\mathbf{U}_{k}=$

$$
\mathbf{R}_{k}^{\dagger}\left(\mathbf{H}_{k} \mathbf{G H}_{1}\right)^{\dagger}\left(\mathbf{H}_{k} \mathbf{G} \mathbf{H}_{1} \mathbf{R}_{\mathbf{x}}\left(\mathbf{H}_{k} \mathbf{G} \mathbf{H}_{1}\right)^{\dagger}+\mathbf{T}_{k} \mathbf{R}_{\mathbf{n}_{\mathrm{I}}, k} \mathbf{T}_{k}^{\dagger}\right)^{-1}
$$

by solving the gradient of $\mathrm{E}\left\{\left(\widehat{\mathbf{s}}_{k}-\mathbf{s}_{k}\right)\left(\widehat{\mathbf{s}}_{k}-\mathbf{s}_{k}\right)^{\dagger}\right\}=0$, when each user $k$ employed a linear Minimum Mean Squared Error (MMSE) receiver [13]. In the rest of the paper, we consider that $P_{1}=\operatorname{tr}\left(\mathbf{R}_{\mathbf{x}}\right)$ and $P_{\mathrm{I}, k}=\operatorname{tr}\left(\mathbf{R}_{\mathrm{I}, k}\right)$, where $P_{1}$ is the average transmit power of the $\mathrm{SN}$ and $P_{\mathrm{I}, k}$ is the average power of each interference signal.

\section{ENHANCED MU MIMO AF PRECODING SCHEMES FOR OCI MITIGATION}

We have recently proposed in [8] two algorithms for solving the optimization problem of (4) in absence of OCI, i.e. when $\mathbf{R}_{\mathbf{n}_{\mathrm{I}}, k}=\sigma_{2, k}^{2} \mathbf{I}_{r_{k}}$. In the following, we revisit our work and enhance our AF-CBD and AF-CGSDL algorithms for mitigating the effect of OCI while maximizing the sum-rate expression in (3).

Instead of using centralized optimization that requires cooperation between cells, we only require the knowledge of the interference plus noise covariance matrix of each user for mitigating the effect of OCI. The latter assumption is more practical because each covariance matrix can be estimated by each user, without requiring synchronization, extra pilot symbols or training sequence, as it is the case in multi-cell cooperation. We also assume as in our AF-CBD and AFCGSDL schemes that the CSI of the SN-RN link, i.e. $\mathbf{H}_{1}$, and the CSI of all the RN-DN links, i.e. $\mathbf{H}_{2, k}$, are known at 
the RN. Since $\mathbf{H}_{1}$ is known at the RN, it can be decomposed via singular valued decomposition (SVD) as $\mathbf{H}_{1}=\mathbf{U} \hat{\boldsymbol{\Lambda}}^{\frac{1}{2}} \mathbf{V}^{\dagger}$ where $\mathbf{U} \in \mathbb{C}^{q \times q}$ and $\mathbf{V} \in \mathbb{C}^{n \times n}$ are unitary matrices, and $\widehat{\Lambda}$ is a $q \times n$ rectangular diagonal matrix. Moreover, $\boldsymbol{\Lambda}=\widehat{\boldsymbol{\Lambda}}^{\frac{1}{2}} \widehat{\boldsymbol{\Lambda}}^{\frac{1}{2} \dagger}$ is a $q \times q$ diagonal matrix with diagonal elements $\lambda_{i} \in \mathbb{R}_{+}$. We also assume that the knowledge of $\mathbf{V}$ is known at the SN and define $\mathbf{R}_{k}$ as

$$
\mathbf{R}_{k}=\widetilde{\mathbf{R}}_{k} \mathbf{V},
$$

where $\widetilde{\mathbf{R}}_{k}=\operatorname{diag}\left(\sqrt{\mathbf{p}_{k}}\right)$ is a $n \times n$ diagonal matrices and $\mathbf{p}_{k}=\left[\begin{array}{lll}\mathbf{0}^{1 \times \alpha_{k}} & \left\{p_{\alpha_{k}+1}, \ldots, p_{\alpha_{k}+l_{k}}\right\} & \mathbf{0}^{1 \times\left(n-\alpha_{k+1}\right)}\end{array}\right]$.

\section{A. Channel block-diagonalization based method}

The matrix $\mathbf{R}_{\mathbf{n}_{\mathrm{I}}, k}$ is clearly a Hermitian positive definite matrix and, thus, it can be decomposed as $\mathbf{R}_{\mathbf{n}_{\mathrm{I}}, k}=\mathbf{D}_{k} \mathbf{D}_{k}^{\dagger}$ by using Cholesky decomposition. Hence, (3) can be re-expressed as

$$
\Sigma_{\mathbf{r}}=\frac{1}{2} \sum_{k=1}^{K} \log _{2}\left|\frac{\sigma_{2, k}^{2} \mathbf{I}_{r_{k}}+\mathbf{H}_{k} \mathbf{G R}_{\mathbf{y}_{1}} \mathbf{G}^{\dagger} \mathbf{H}_{k}^{\dagger}}{\sigma_{2, k}^{2} \mathbf{I}_{r_{k}}+\mathbf{H}_{k} \mathbf{G R}_{\mathbf{n}_{1}, k} \mathbf{G}^{\dagger} \mathbf{H}_{k}^{\dagger}}\right|
$$

by setting $\mathbf{T}_{k}=\sigma_{2, k} \mathbf{D}_{k}^{-1}$ in (3), which is then equivalent to the expression of the relay link sum-rate in absence of OCI, i.e. equation (5) of [8], but with $\mathbf{H}_{k}$ instead of $\mathbf{H}_{2, k}$. In other words, the effect of OCI has simply been transferred into the equivalent channel $\mathbf{H}_{k}=\mathbf{T}_{k} \mathbf{H}_{2, k}$ by setting $\mathbf{T}_{k}=\sigma_{2, k} \mathbf{D}_{k}^{-1}$ at each DN. Consequently, instead of feedbacking $\mathbf{H}_{2, k}$ in absence of OCI, each DN must feedback $\mathbf{H}_{k}$ in presence of OCI.

In CBD [14], the precoder design is performed in two phases; in the first phase, the structure of the precoder is designed for cancelling intra-cell interference via blockdiagonalization of the multi-user channel. In the second phase, optimal power allocation is performed. In our AF-ECBD, we follow the same process to design the precoder $\mathbf{G}$ at the RN. First, we define the precoder structure of $\mathbf{G}$ as

$$
\mathbf{G}=\mathbf{W} \widetilde{\mathbf{G}} \mathbf{U}^{\dagger},
$$

where $\mathbf{W}=\left[\mathbf{W}_{1}, \mathbf{W}_{2}, \ldots, \mathbf{W}_{K}\right], \mathbf{W}_{k} \in \mathbb{C}^{q \times l_{k}}$, and $\widetilde{\mathbf{G}}=\operatorname{diag}(\sqrt{\mathbf{g}}), \mathbf{g}=\left\{g_{1}, \ldots, g_{q}\right\}$. Each matrix $\mathbf{W}_{k}$ is then designed by ensuring that $\mathbf{H}_{j} \mathbf{W}_{k}=$ $\mathbf{0}, \forall j \neq k$, i.e. the intra-cell interference is nulled. Let $\overline{\mathbf{H}}_{k}=\left[\mathbf{H}_{1}^{\dagger}, \ldots, \mathbf{H}_{k-1}^{\dagger}, \mathbf{H}_{k+1}^{\dagger}, \ldots, \mathbf{H}_{K}^{\dagger}\right]^{\dagger}$ be the complementary channel of user $k, \mathbf{Y}_{k}$ be a matrix of rank $\rho_{k}$ that contains the $q$ right-singular vectors of $\overline{\mathbf{H}}_{k}$ and $\mathbf{Y}_{k,\left[\rho_{k}+1: q\right]}$ contains the last $q-\rho_{k}$ columns of $\mathbf{Y}_{k}$. In addition, let $\mathbf{Z}_{k}$ be a matrix that contains the $q-\rho_{k}$ right-singular vectors of $\mathbf{H}_{k} \mathbf{Y}_{k,\left[\rho_{k}+1: q\right]}$ and $\mathbf{Z}_{k,\left[1: l_{k}\right]}$ contains the first $l_{k}$ columns of $\mathbf{Z}_{k}$. Then, $\mathbf{W}_{k}$ is simply defined as

$$
\mathbf{W}_{k}=\mathbf{Y}_{k,\left[\rho_{k}+1: q\right]} \mathbf{Z}_{k,\left[1: l_{k}\right]}
$$

for ensuring that the intra-cell interference is nulled. Notice that each user transmit a message of length $l_{k}$ that is transmitted over $l_{k}$ streams. Thus, the condition $\rho_{k}+l_{k} \leq q$ must hold for applying CBD. Otherwise, stream selection must be performed prior to the precoding. Moreover, if $K>q$ then user selection must be performed prior to the precoding. Inserting (6) and (8) into (7), the latter simplifies as

$$
\Sigma_{\mathbf{r}}(\mathbf{g})=\frac{1}{2} \sum_{k=1}^{K} \prod_{i=1}^{l_{k}} \log _{2}\left(\frac{1+g_{u} \omega_{u}\left(1+\lambda_{u} p_{u}\right)}{1+g_{u} \omega_{u}}\right),
$$

where $u=\alpha_{k}+i$ and $\omega_{u}$ is the $i$-th nonnegative eigenvalue of $\mathbf{H}_{k} \mathbf{Y}_{k,\left[\rho_{k}+1: q\right]} \mathbf{Y}_{k,\left[\rho_{k}+1: q\right]}^{\dagger} \mathbf{H}_{k}^{\dagger}$. The optimal power allocation is then obtained by solving the following optimization problem

$$
\max _{\mathbf{g}} \Sigma_{\mathbf{r}}\left(\text { g) s.t. } g_{u} \geq 0 ; \sum_{k=1}^{K} \sum_{i=1}^{l_{k}} g_{u}\left(1+\lambda_{u} p_{u}\right) \leq P_{2},\right.
$$

which is equivalent to the optimization problem in [4] when $K=1$ and $l_{1}=r_{1}$. This convex problem can directly be solved by following a similar approach as in [4].

In a nutshell, the OCI has first been mitigated by using the postcoding matrix $\mathbf{T}_{k}$ and then the MU MIMO relay channel has been block-diagonalized and thus transformed into $K$ independent MIMO relay channels by using the knowledge on $\mathbf{H}_{1}$ and all the $\mathbf{H}_{k}$ at the RN.

\section{B. Constrained gradient search based method}

The $\mathbf{G}$ matrix structure in (8) turns out to be optimal in the single user case [4]. However, it has recently been reported in [7] that it is no more the case in the MU context. Therefore, instead of decomposing the precoding at the RN in two phases as in the AF-ECBD, we can use a CGS algorithm for finding a $\mathbf{G}$ matrix that maximizes (3). The postcoding matrix $\mathbf{T}_{k}=$ $\sigma_{2, k} \mathbf{D}_{k}^{-1}$ is first used at each DN for removing the OCI such that (3) turns into (7) and then the same CGS algorithm as in [8] is applied, but where the gradient of $\Sigma_{\mathbf{r}}$ is given by

$$
\begin{aligned}
\frac{\partial \Sigma_{\mathbf{r}}}{\partial \mathbf{G}} & =\frac{1}{\ln (2)} \sum_{k=1}^{K} \mathbf{H}_{k}^{\dagger}\left(\sigma_{2, k}^{2} \mathbf{I}_{r_{k}}+\mathbf{H}_{k} \mathbf{G R}_{\mathbf{y}_{1}} \mathbf{G}^{\dagger} \mathbf{H}_{k}^{\dagger}\right)^{-1} \mathbf{H}_{k} \mathbf{G R}_{\mathbf{y}_{1}} \\
& -\mathbf{H}_{k}^{\dagger}\left(\sigma_{2, k}^{2} \mathbf{I}_{r_{k}}+\mathbf{H}_{k} \mathbf{G} \mathbf{R}_{\mathbf{n}_{1}, k} \mathbf{G}^{\dagger} \mathbf{H}_{k}^{\dagger}\right)^{-1} \mathbf{H}_{k} \mathbf{G R}_{\mathbf{n}_{1}, k}
\end{aligned}
$$

since $\partial \ln \left|\mathbf{I}+\mathbf{X Y \mathbf { X } ^ { \dagger }}\right| / \partial \mathbf{Y}=2\left(\mathbf{I}+\mathbf{X Y X ^ { \dagger }}\right)^{-1} \mathbf{X Y}$ if $\mathbf{Y}$ is an Hermitian matrix. This algorithm has a greater computational complexity than the AF-ECBD as indicated by the numerical computational complexity analysis in Fig. 3. In comparison with a standard gradient search algorithm, extra computation is needed to ensure that the searched $\mathbf{G}$ matrices are always within the search space, which slightly increases the complexity.

\section{RESUlTS}

In this section the performances of our OCI-aware precoding techniques for MU MIMO AF, i.e. AF-ECBD and AFECGSDL, are compared against each others and against the AF-CBD and AF-CGSDL schemes of [8] in terms of sumrate and computational complexity when $\mathrm{OCI}$ is present. In addition, the performances of the AF-CBD and AF-CGSDL schemes are also plotted in absence of OCI, as benchmark results.

In our simulations, we define the SNR of the SN-RN link as $\gamma_{1}=P_{1} / \sigma_{1}^{2}$, the SNR of the $k$-th RN-DN link 


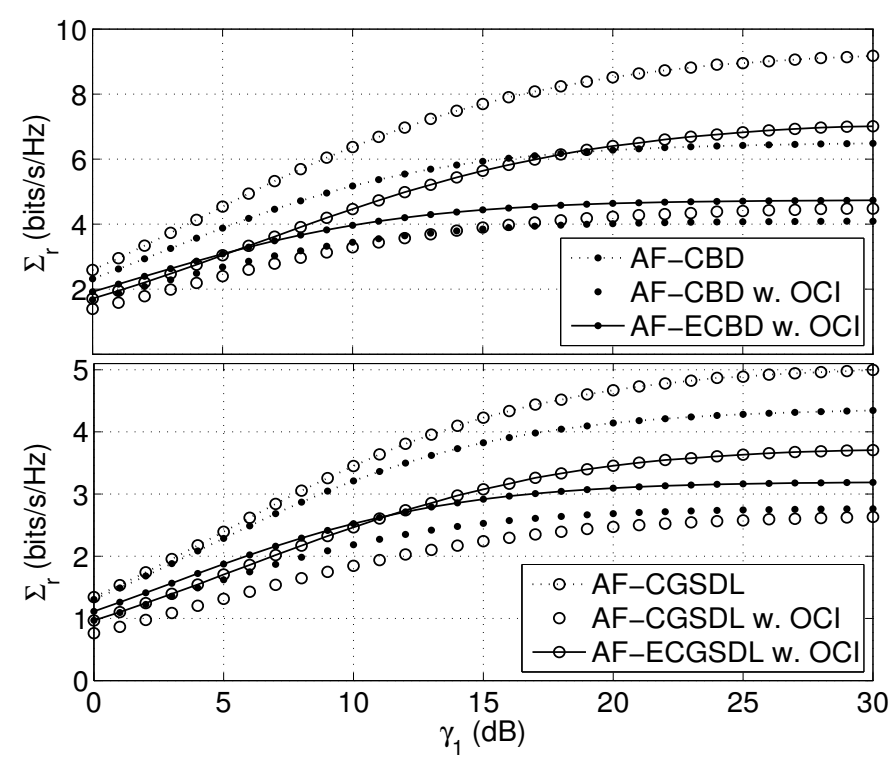

Fig. 2. Sum-rate performances of our OCI-aware precoding schemes for $n=q=4, K=2$ (lower graph) and $n=q=8, K=4$ (upper graph) when $\gamma_{2, k}=10 \mathrm{~dB}, \gamma_{\mathrm{I}, k}=20 \mathrm{~dB}, r_{k}=l_{k}=2$, and $n_{\mathrm{I}, k}=1$.

as $\gamma_{2, k}=P_{2} / \sigma_{2, k}^{2}$ and the interference to noise ratio of the $k$-th user as $\gamma_{\mathrm{I}, k}=P_{\mathrm{I}, k} / \sigma_{2, k}^{2}$, where we consider that $\sigma_{1}^{2}=\sigma_{2, k}^{2}=1, \forall k \in[1, \ldots, K]$. We assume an equal gain power allocation at the $\mathrm{SN}$ and set $p_{\alpha_{k}+j}=P_{1} / l$, $\forall j \in\left[1, \ldots, l_{k}\right]$ and $\forall k \in[1, \ldots, K]$. We also assume as in [11] that $\mathbf{R}_{\mathrm{I}, k}=\left(P_{\mathrm{I}, k} / n_{\mathrm{I}, k}\right) \mathbf{I}_{n_{\mathrm{I}, k}}$. This assumption is not typical but it allows us to evaluate the capacity degradation due to OCI in the worst case scenario. In addition, we assume a single-tap i.i.d. Rayleigh fading channel between any of the nodes and consider $5 \times 10^{3}$ realisations of each channel for evaluating the sum-rate $\Sigma_{\mathbf{r}}$. The CGS algorithm of [8], i.e. Algorithm 1, has been modified and utilized to plot the results for our AF-ECGSDL method; the parameter $\epsilon$, which is used for fine-tuning the trade-off between accuracy and complexity, has been set to $\epsilon=10^{-4}$. Finally, the power allocation in (11) for the AF-ECBD method has been performed by considering the following sorting of the elements of $\Lambda$. Let $\bar{\lambda}$ be the vectors of elements $\lambda_{i}$ that are sorted in descending order such that $\bar{\lambda}_{1} \geq \bar{\lambda}_{2} \geq \ldots \geq \bar{\lambda}_{q}$. Moreover, let ind $=\left[1, K+1, \ldots,\left(l_{1}-\right.\right.$ 1) $\left.K+1,2, K+2, \ldots,\left(l_{2}-1\right) K+2, \ldots, K, 2 K, \ldots, l_{k} K\right]$ be a set of indices, then we have set $\lambda_{i}=\bar{\lambda}_{\text {ind }_{i}}$.

In Fig. 2, we compare the sum-rate performances of our AF-ECBD and AF-ECGSDL schemes for $n=q=4, K=2$ and $n=q=8, K=4$, in the lower and upper parts of Fig. 2, respectively. In addition, we set $\gamma_{2, k}=10 \mathrm{~dB}, \gamma_{\mathrm{I}, k}=20 \mathrm{~dB}$, $r_{k}=l_{k}=2$ and $n_{\mathrm{I}, k}=1, \forall k \in[1, \ldots, K]$. The results first indicate that in absence of OCI, the AF-CGSDL outperforms the AF-CBD scheme and the performance difference between these two schemes increases as the size of the MU MIMO AF system, i.e. either the number of antennas or users, increases, as already pointed out in [8]. In presence of OCI, the results show that the performances of both schemes drop dramatically and that the AF-CBD outperforms the AF-CGSDL scheme in

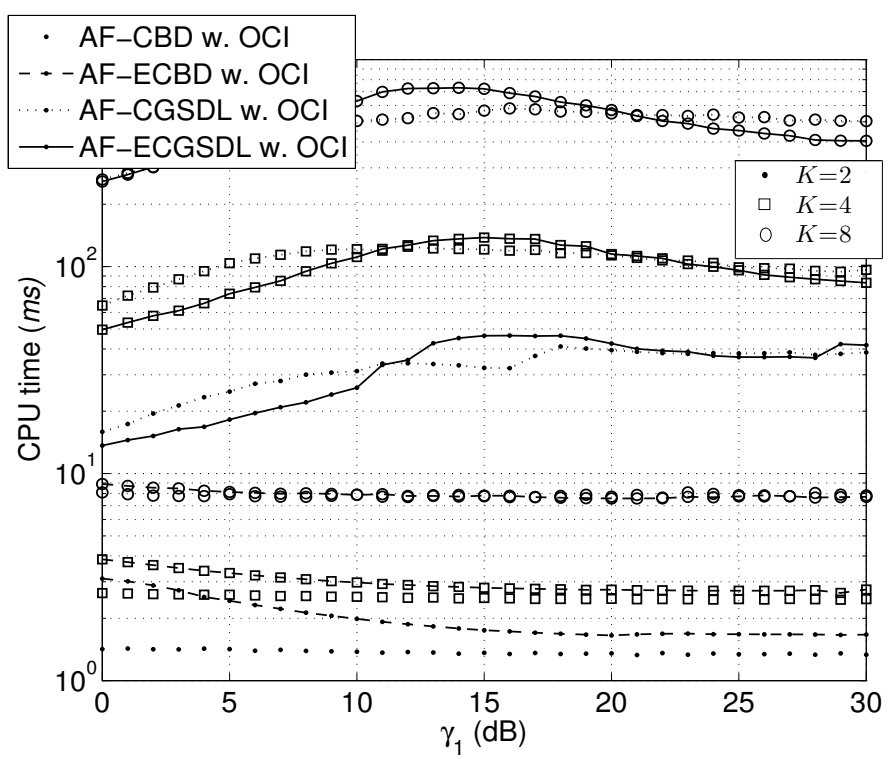

Fig. 3. Computational complexity of our OCI-aware precoding schemes for $n=q=4, K=2, n=q=8, K=4$ and $n=q=16, K=8$ when $\gamma_{2, k}=10 \mathrm{~dB}, \gamma_{\mathrm{I}, k}=20 \mathrm{~dB}, r_{k}=l_{k}=2$, and $n_{\mathrm{I}, k}=1$.

the case of $K=2$ and for low $\gamma_{1}$ values in the case of $K=4$. Then, we can remark that the effect of OCI is clearly mitigated by using our novel OCI-aware precoding schemes and that the mitigation gain increases as the size of the MU MIMO AF system increases; for instance, the AF-ECGSDL method outperforms the AF-CGSDL method by 1 and $2.5 \mathrm{bits} / \mathrm{s} / \mathrm{Hz}$ in the case of $K=2$ and 4, respectively, when $\gamma_{1}=30 \mathrm{~dB}$. In addition, the AF-ECGSDL method mitigates better the OCI than the AF-ECBD method at high SNRs, whereas it is the contrary at low SNRs.

In Fig. 3, we complement our results of Fig. 2 by comparing the computational complexity of our two schemes for the same settings as in Fig. 2 plus the case where $n=q=16$ and $K=8$. We utilize the average CPU execution time of each algorithm in milliseconds $(m s)$ as a comparison metric. The AF-CBD and AF-CGSDL schemes have obviously the same execution time in absence or presence of OCI, since OCI is not incorporated in their precoder structures and, therefore, we omit the absence of CSI case in this graph. The results clearly show that the AF-ECBD scheme is less computationally demanding that the AF-ECGSDL scheme and that the computational complexity of the two schemes clearly increases as the size of the MU MIMO AF system increases; it increases in a faster way for the AF-ECGSDL scheme such that the AF-ECBD scheme is at least 10 times and about 50 times less computationally demanding than the AFECGSDL scheme in the $K=2$ and $K=8$ cases, respectively. Moreover, by comparing the AF-CBD with the AF-ECBD scheme, it appears that the AF-ECBD scheme requires extra computational complexity, mainly at low SNRs, for mitigating the OCI when the size of the MU MIMO AF system is small. As the size increases, the two schemes exhibit the same computational complexity. On the contrary, the AF-ECGSDL 


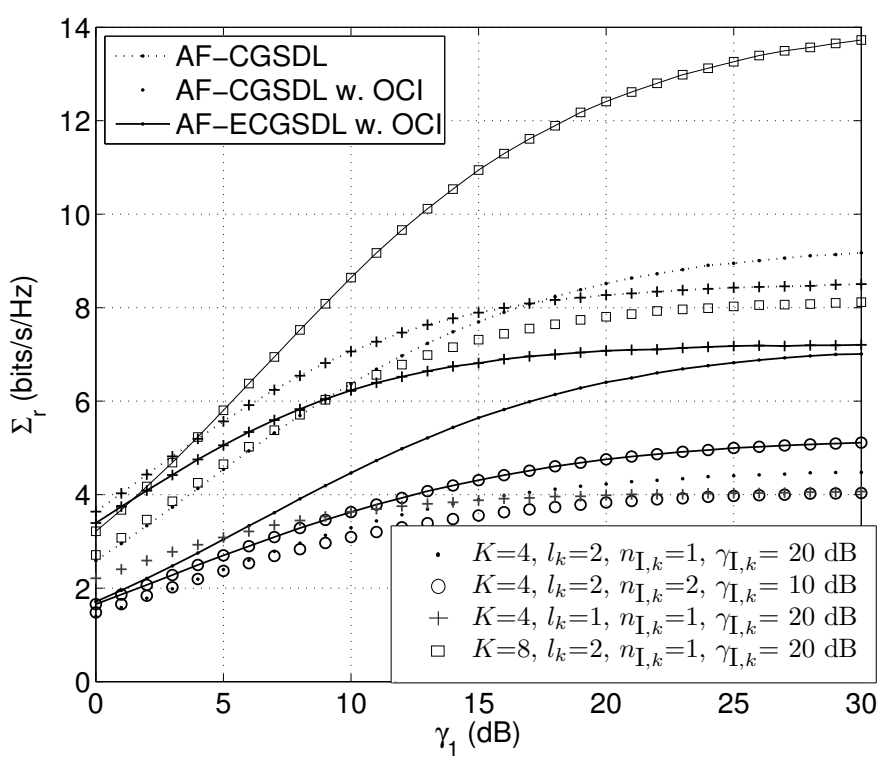

Fig. 4. Sum-rate performances of our OCI-aware precoding schemes for $n=q=4, K=2$ and $n=q=8, K=4$ with various values of $l_{k}, \gamma_{\mathrm{I}, k}$ and $n_{\mathrm{I}, k}$ when $\gamma_{2, k}=10 \mathrm{~dB}$ and $r_{k}=2$.

is less computational demanding than the AF-CGSDL scheme at low SNRs.

In Fig. 4, we compare the AF-CGSDL and AF-ECGSDL sum rate performances for $n=q=8, K=4$ and $n=q=16$, $K=8$, and various values of $l_{k}, \gamma_{\mathrm{I}, k}$ and $n_{\mathrm{I}, k}$ when $\gamma_{2, k}=10$ $\mathrm{dB}$ and $r_{k}=2, \forall k \in[1, \ldots, K]$. In the case of $K=4$, the results show that for different OCI settings, i.e. different values of $n_{\mathrm{I}, k}$ and $P_{\mathrm{I}, k}$, the AF-CGSDL scheme provides roughly the same sum-rate performances, which are about half of those obtained in absence of OCI. The effect of OCI can be mitigated by using our AF-ECGSDL, especially when $l_{k}=n_{\mathrm{I}, k}=1$. In this case, each user transmit by using only one stream and the OCI occupy one stream as well; however, $r_{k}=2$ and, hence, each user has two degrees of freedom for accommodating at the same time the user data and the OCI, which allows the AF-ECGSDL scheme to greatly reduces the effect of OCI. In the case of $l_{k}=2$ and $n_{\mathrm{I}, k}=1$ or 2 , the user data and OCI cannot be decoupled and the performance of the AF-ECGSDL scheme is worse than in the previous case, especially when $n_{\mathrm{I}, k}=2$, i.e the two user data streams are affected by OCI. Finally, the results obtained for $K=8$ confirm that the sumrate performance improvement generated by our AF-ECGSDL scheme increases with the size of the MU MIMO AF system.

\section{CONCLusions}

In this paper, we have introduced two novel precoding schemes for the DL of MU MIMO AF communication system that are designed to maximize the sum-rate performance of cell-edge users in presence of OCI. We have extended our work in [8] to the multi-cell scenario by incorporating the effect of OCI in our precoding structure at the RN. We have formulated a sum-rate expression for the MU MIMO AF communication system in presence of OCI and have utilized it for designing our precoding schemes. These schemes are practical in th sense that they do not require multi-cell cooperation for mitigating the OCI nor dirty paper coding for mitigating intra-cell interference. They only require the knowledge of the SN-RN and RN-DN link CSI and the interference plus noise covariance matrix of each cell-edge user to be available at the RN. Results have demonstrated that our OCI-aware schemes outperform non OCI-aware schemes in presence of OCI. Among them, the AF-ECBD scheme is low-complexity but provides low sum-rate performances, whereas, the AFECGSDL scheme provides far better performances but at the expense of a higher computational complexity. In our future works, we will design a joint precoding scheme at the SN and $\mathrm{RN}$ for mitigating OCI when the direct link is active, as well as compare the energy efficiency of this scheme against multi-cell cooperation.

\section{ACKNOWLEDGMENT}

The research leading to these results has received funding from the European Commission's Seventh Framework Programme FP7/2007-2013 under grant agreement $n^{\circ} 247733$ project EARTH.

\section{REFERENCES}

[1] A. Sendonaris, E. Erkip, and B. Aazhang, "User Cooperation Diversity Part I \& II- System Description / Implementation Aspects and Performance Analysis," IEEE Trans. Commun., vol. 51, pp. 1927-1948, Nov. 2003.

[2] A. Nosratinia, T. E. Hunter, and A. Hedayat, "Cooperative Communication in Wireless Networks," IEEE Commun. Mag., vol. 42, pp. 74-80, Oct. 2004.

[3] J. N. Laneman, D. N. C. Tse, and G. W. Wornell, "Cooperative Diversity in Wireless Networks: Efficient Protocols and Outage Behavior," IEEE Trans. Inform. Theory, vol. 50, pp. 3062-3080, Dec. 2004.

[4] O. Muñoz-Medina, J. Vidal, and A. Agustn, "Linear Transceiver Design in Nonregenerative Relays with Channel State Information," IEEE Trans. Signal Processing, vol. 55, pp. 2593-2604, June 2007.

[5] L. Weng and R. Murch, "Multi-user MIMO Relay System with SelfInterference Cancelation," in Proc. IEEE WCNC, (Hong-Kong, China), Mar. 2007.

[6] C. Chae, T. Tang, R. Health, and S. Cho, "MIMO Relaying with Linear Processing for Multi-user Transmission in Fixed Relay Networks," IEEE Trans. Inform. Theory, vol. 56, pp. 727-738, Feb. 2008.

[7] Y. Yu and Y. Hua, "Power Allocation for a MIMO Relay System with Multiple-Antenna Users," IEEE Trans. Signal Processing, vol. 58, pp. 2823-2835, May 2010.

[8] F. Héliot, R. Hoshyar, and R. Tafazolli, "Power Allocation for the Downlink of Nonregenerative Cooperative Multi-User MIMO Communication System," in Proc. IEEE PIMRC, (Istanbul, Turkey), Sept. 2010.

[9] S. Ye and R. S. Blum, "Optimized Signaling for MIMO Interference Systems with Feedback," IEEE Trans. Signal Processing, vol. 51, pp. 2839-2848, Nov. 2003.

[10] J. G. Andrews, W. Choi, and R. W. Heath, "Overcoming Interference in Spatial Multiplexing MIMO Wireless Networks," IEEE Wireless Commun. Mag., vol. 14, pp. 95-104, Dec. 2007.

[11] S. Shim, J. S. Kwak, R. W. Heath, and J. G. Andrews, "Block Diagonalization for Multi-User MIMO with Other-Cell Interference," IEEE Trans. Wireless Commun., vol. 7, pp. 2671-2681, July 2008.

[12] T. M. Cover and J. A. Thomas, Elements of Information Theory. NewYork, USA: ed. Wiley, 1991.

[13] A. J. Tenenbaum and R. S. Adve, "Joint Multiuser Transmit-Receive Optimization Using Linear Processing," in Proc. IEEE ICC '04, (Paris, France), pp. 588-592, June 2004.

[14] Q. H. Spencer, A. L. Swindlehurst, and M. Haardt, "Zero-Forcing Methods for Downlink Spatial Multiplexing in Multiuser MIMO Channels," IEEE Trans. Signal Processing, vol. 52, pp. 461-471, Feb. 2004. 